\title{
The dark cloud TGU H994 P1 (LDN 1399, LDN 1400, and LDN 1402): Interstellar extinction and distance ${ }^{\star}$
}

\author{
V. Straižys ${ }^{1}$, V. Čepas ${ }^{1}$, R. P. Boyle ${ }^{2}$, U. Munari ${ }^{3}$, J. Zdanavičius ${ }^{1}$, M. Maskoliūnas ${ }^{1}$, \\ A. Kazlauskas ${ }^{1}$, and K. Zdanavičius ${ }^{1}$
}

\author{
${ }^{1}$ Institute of Theoretical Physics and Astronomy, Vilnius University, Goštauto 12, 01108 Vilnius, Lithuania \\ e-mail: vytautas.straizys@tfai.vu.lt \\ 2 Vatican Observatory Research Group, Steward Observatory, Tucson, AZ 85721, USA \\ 3 INAF Astronomical Observatory of Padova, 36012, Asiago (VI), Italy
}

Received 4 August 2015 / Accepted 5 October 2015

ABSTRACT

\begin{abstract}
The results of CCD photometry in the seven-colour Vilnius system, for about 1000 stars down to $V=20$ mag and their twodimensional spectral types, are used to investigate the interstellar extinction in a 1.5 square degree area in the direction of the dark cloud TGU H994 P1 (LDN 1399, LDN 1400 and LDN 1402) in Camelopardalis. Photometric classification of 18 brightest stars down to $V=12 \mathrm{mag}$ was verified by the spectra obtained with the $1.22 \mathrm{~m}$ telescope of the Asiago Observatory. The interstellar extinction run with distance is investigated with the results of photometry in the Vilnius system, and 504 red clump giants, identified by combining the results of infrared photometry from the 2MASS and WISE surveys. A possible distance of $140 \pm 11 \mathrm{pc}$ to the TGU H994 P1 cloud seems to be acceptable. Alternative distances of the cloud are discussed. The complex of the Camelopardalis clouds probably has a considerable depth along the line of sight, similar to that observed in the Taurus-Auriga complex. The maximum extinction $A_{V}$ in the dark filaments is found to be about 6.5 mag.
\end{abstract}

Key words. stars: fundamental parameters - ISM: clouds - ISM: individual: TGU H994 P1, LDN 1399, LDN 1400, LDN 1402

\section{Introduction}

A few years ago Straižys \& Laugalys $(2007,2008)$ described a ring-like concentration of dust clouds located at the border of the Perseus, Camelopardalis, and Auriga constellations, which is perfectly seen in the Dobashi et al. (2005) atlas of dark clouds. The ring has a diameter of $\sim 8^{\circ}$ and is centred on the open cluster NGC 1528. Trying to test physical reality of this dust ring, we decided to investigate distances to its clouds and their extinction properties. The method of investigation is based on photometry of stars in the Vilnius seven-colour system, determination of their spectral and luminosity classes, reddenings, extinctions and distances. The first three catalogues of photometric data and spectral classification in the clouds TGU H1000, TGU H942, and TGU H994 were published by Zdanavičius et al. (2010) and Čepas et al. (2013a,b). In this paper, we present the investigation of interstellar reddening in the clump P1 of the TGU H994 cloud located in the northern side of the ring.

A curved $\sim 5^{\circ}$-long chain of dark clouds, located westwards from the triple star 1 Cam (DL Cam, HD 28446), was described in the Barnard (1927) catalogue and atlas of dark clouds (clouds B8, B9, B11, B12). Barnard writes that this chain of dark clouds breaks up into more or less separate spots, "somewhat resembling those at the east end of the great lane from Rho Ophiuchi". Again, dust clumps of this chain appear in the catalogues of dark clouds by Lynds (1962), Taylor et al. (1987), Dutra \& Bica (2002), Dobashi et al. (2005), and Dobashi (2011).

\footnotetext{
* Full Table 2 is only available at the CDS via anonymous ftp to cdsarc.u-strasbg. fr $(130.79 .128 .5)$ or via http://cdsarc.u-strasbg.fr/viz-bin/qcat?J/A+A/585/A31
}

Unfortunately, it is not easy to cross-identify separate clouds in different catalogues since their boundaries usually remain indefinite. The present investigation covers the clump P1 of the cloud TGU H994 (Dobashi et al. 2005), which includes the Barnard cloud B11 and the Lynds clouds LDN 1399, LDN 1400, and LDN 1402.

Until now these clouds were investigated in the molecular bands: CO (Dickman 1975; Snell 1981; Myers \& Benson 1983; Taylor et al. 1987; Dame et al. 1987, 2001; Park et al. 2004; Wu et al. 2012), $\mathrm{H}_{2} \mathrm{CO}$ (Dieter 1973; Minn \& Greenberg 1973; Sandqvist et al. 1988; Clark 1991), $\mathrm{NH}_{3}$ (Myers \& Benson 1983; Benson \& Myers 1989; Clark 1991), CS, and $\mathrm{N}_{2} \mathrm{H}$ (Lee \& Myers 1999). The local standard of rest (LSR) velocity of these clouds is $2-4 \mathrm{~km} \mathrm{~s}^{-1}$. Myers \& Benson (1983) identified a group of visually opaque gas and dust condensations in the cloud LDN 1400 and the surrounding clouds, named cores, which are the supposed progenitors of young stars. Some of these objects are included in the catalogue of optically selected cores by Lee \& Myers (1999). They were also observed with the Planck and Herschel satellites in the submillimeter and the far-infrared spectral regions (Juvela et al. 2012). About 30 far-infrared sources in the area of LDN 1400 and other nearby clouds were identified in the IRAS point-source catalogue by Clark (1991). Harjunpää et al. (1999) investigated polarization of stars in some directions of the LDN 1400 complex.

Distances to the chain clouds are known with a very low accuracy. The first estimate of their distance was probably published by Snell (1981). As the author explains, the estimate was done from the $A_{V}$ vs. $d$ plot constructed for the stars with known $B, V$ photometry and MK spectral types in 
a $20^{\circ} \times 20^{\circ}$ region centred on the cloud. Although a value of $d=170$ pc has been attributed to a small cloud in the group (LDN 1407, the size $8^{\prime} \times 8^{\prime}$ ), actually this distance can be related to a much larger cloud complex. However, this distance should be of low accuracy since the stars with $B, V$ photometry and MK spectral types in this region are rather scarce. This distance value (with nothing better available) has been used for decades in many investigations of the dust and molecular clouds in the whole chain. For the right group of the chain clouds (around LDN 1394), a distance value of $0.66 \mathrm{kpc}$ was obtained by Juvela et al. (2012) applying a statistical method based on the Besancon stellar population synthesis model and the 2MASS photometry data ${ }^{1}$.

In several directions of the Camelopardalis dark clouds, the interstellar extinction was investigated by Zdanavičius et al. (1996, 2001, 2005), Zdanavičius \& Zdanavičius (2002a,b, 2005) using seven-colour photometry in the Vilnius system and photometric classification of stars in spectral and luminosity classes. A common property of all areas is the extinction rise at 120$150 \mathrm{pc}$, reaching $A_{V}$ about $1.5-2.5 \mathrm{mag}$ at $1 \mathrm{kpc}$. Thus, the Camelopardalis clouds are located almost at the same distance as the Taurus-Auriga star-forming region; see also the review article in the "Handbook of Star Forming Regions" by Straižys \& Laugalys (2008).

Distances to dust clouds can be also estimated from interstellar line intensities observed in the spectra of foreground stars and the stars immersed in the cloud, usually of spectral classes B and A. The most detailed investigations of interstellar $\mathrm{Na}$ I D lines and $\mathrm{Ca}$ II $\mathrm{H}+\mathrm{K}$ lines in the solar vicinity were published by Welsh et al. (1994, 2010), Sfeir et al. (1999), Vergely et al. (2001, 2010), Lallement et al. (2003, 2014), using the Hipparcos distances. In the general direction of Taurus and Auriga, the concentration of neutral gas is seen in the $80-150 \mathrm{pc}$ distance range that can be associated with the boundary wall of the rarefied local cavity region around the Sun.

The TGU H994 P1 cloud is covered by one of the fields of the $\mathrm{APOGEE}^{2}$ spectral survey of red clump giants (RCGs, Bovy et al. 2014). From 155 RCGs in the field, 30 are located within our area, and the remaining are up to $\sim 2^{\circ}$ to the right and $\sim 1.5^{\circ}$ upwards from the investigated area. These stars are important tracers of the extinction at large distances, but they are lacking at the distances of cloud LDN 1400.

According to Dame et al. (1987, 2001), column densities and radial velocities of $\mathrm{CO}$ in some directions of the 2 nd galactic quadrant exhibit two distinct layers at $800 \mathrm{pc}$ and $300 \mathrm{pc}$ from the Sun. The more distant layer, with the LSR velocity $-12 \mathrm{~km} \mathrm{~s}^{-1}$, is related to the chain of $\mathrm{OB}$ associations at the outer edge of the Local Arm belonging to the Gould Belt. The closer layer with LSR velocity close to zero is related to the expanding gas ring discovered by Lindblad et al. (1973) from observations of neutral hydrogen; see also Lindblad (1974). In the direction of Cam-Per-Tau the Lindblad Ring can be also related to the Per OB3 (or $\alpha$ Per) association $(d=177 \mathrm{pc}$ ) and the CasTau moving group of B-type stars ( $d$ from 125 to $300 \mathrm{pc}$ ); the distances are from de Zeeuw et al. (1999).

We found only four stars with the Hipparcos parallaxes in the investigated area: No. $13=$ HIP 20327 (A0 V, $d=318$ pc), No. $341=$ HIP 20655 (A1 IV-V, $d=256$ pc), No. $420=$ HIP $20720(\mathrm{~B} 8 \mathrm{~V}, d=3.5 \mathrm{kpc})$, and HD $28097=$ HIP 20910

\footnotetext{
1 In Table 1 of Juvela et al. (2012), the identification of the cloud LDN 1400 is not correct. It is located in the same area, called G150.47+3.93, as the LDN 1399 cloud.

2 Apache Point Observatory Galactic Evolution Experiment.
}

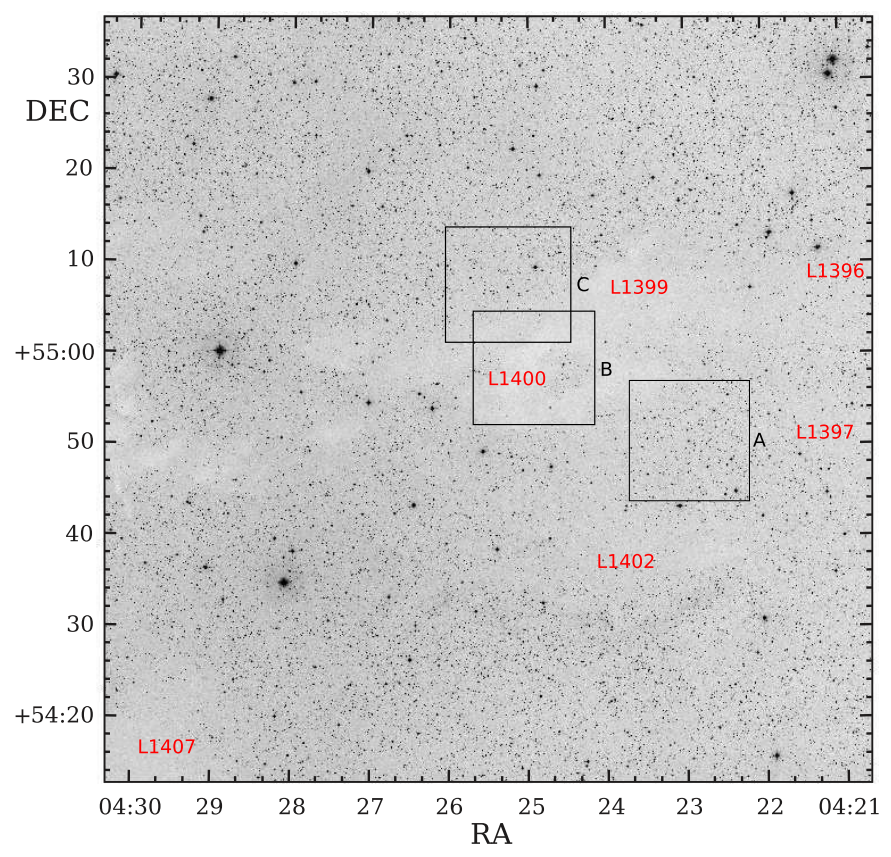

Fig. 1. Map with the observed area in the red filter (DSS1 Red from the SkyView Virtual Observatory). The three smaller squares were observed with the VATT telescope to fainter magnitudes.

(F7 V,$d=81.5 \mathrm{pc}$, too bright for our CCD photometry). The star numbers are from the catalogue Čepas et al. (2013b), hereafter Paper I. The accuracy of parallaxes of the first three stars is rather low.

\section{Photometric data and spectral types}

Exposures of the area (Fig. 1) in the Vilnius seven-colour system were obtained in October of 2010 with the Maksutovtype $35 / 51 \mathrm{~cm}$ telescope of the Molètai Observatory in Lithuania, equipped with a CCD camera. The area covers $1.5 \mathrm{deg}^{2}$ with the centre at RA $(\mathrm{J} 2000)=04^{\mathrm{h}} 25.5^{\mathrm{m}}$, Dec $(\mathrm{J} 2000)=+54^{\circ} 55^{\prime}, \ell=150.53^{\circ}, b=+3.96^{\circ}$. More details about the observations and their processing are given in Paper I. The Vilnius photometric system UPXYZVS with the mean wavelengths $345,374,405,466,516,544$, and $656 \mathrm{~nm}$ is described in the Straižys (1992) monograph.

The catalogue published in Paper I gives the results of photometry for 727 stars down to $V \approx 17 \mathrm{mag}$. For about $73 \%$ of stars, two-dimensional spectral types (spectral and luminosity classes) were determined applying two classification codes based on interstellar reddening-free $Q$-parameters. One of these codes, COMPAR, applies a comparison of $Q$-parameters of stars in the area and individual standard stars (Straižys et al. 2013). The second code, now renamed to KLASQ, is described in Milašius et al. (2013). Instead of standard stars with known MK spectral types, it applies the intrinsic colour indices calibrated in MK types. In the present investigation, the stars of Paper I were reclassified anew with a modernized version of the second code (KLASQ), therefore, for some stars, new spectral and luminosity classes show small differences in comparison with Paper I.

Additionally, for the classification of fainter stars down to $V \approx 20 \mathrm{mag}$, we applied Vilnius photometry in three $12^{\prime} \times 12^{\prime}$ areas located at the edges and within the LDN 1400 cloud. The areas are framed in Fig. 1 and their central coordinates are given in Table 1 . We obtained CCD observations 
Table 1. Centres of the three $12^{\prime} \times 12^{\prime}$ areas observed with the $1.8 \mathrm{~m}$ VATT telescope (see Fig. 1).

\begin{tabular}{lccr}
\hline \hline Area & RA (2000) & Dec (2000) & $\begin{array}{r}\text { Number } \\
\text { of stars }\end{array}$ \\
\hline LDN 1400 A & $04: 22: 58$ & $+54: 51: 24$ & 438 \\
LDN 1400 B & $04: 24: 53$ & $+54: 58: 20$ & 56 \\
LDN 1400 C & $04: 25: 15$ & $+55: 07: 08$ & 370 \\
\hline
\end{tabular}

in the Vilnius system in November and December 2014 and January 2015 with the $1.8 \mathrm{~m}$ VATT telescope of the Vatican Observatory on Mt. Graham, Arizona. In each area we processed about 70 frames obtained with the exposures from 30 min to $5 \mathrm{~s}$. Instrumental colour indices were transformed to the standard system with colour equations obtained from observations of the cluster M67 (Laugalys et al. 2004). The stars measured in Paper I served as zero-point standards of magnitudes and colour indices. The final adjustment of zero points has been done by optimizing the accuracy of photometric classification of a selected set of standard stars in the investigated area. We performed photometric classification of stars with the same COMPAR and KLASQ codes mentioned above, and the results were averaged.

The catalogue of photometric data for 855 stars down to $V \approx 20 \mathrm{mag}$ and the results of photometric two-dimensional classification for about $65 \%$ of them will be accessible at the Strasbourg Data Center. Table 2 gives the beginning of this catalogue for the first ten stars. The columns list the following information: star number, equatorial coordinates J2000.0, magnitude $V$, colour indices $U-V, P-V, X-V, Y-V, Z-V$ and $V-S$, and photometric spectral types in the MK system. Photometric spectral classes are given in lower-case letters to distinguish from spectroscopic classes. The suspected metal-deficient stars are designated as md:, and the metal-rich stars (Am) as mr:. The coordinates are from the PPMXL catalogue (Roeser et al. 2010) rounded to two decimals of time second and to one decimal of arcsecond.

The rms errors of the magnitudes $V$ and colour indices $X-V$, $Y-V, Z-V$ and $V-S$ down to $V=16$ mag are usually lower than $0.02 \mathrm{mag}$, and the errors of $U-V$ and $P-V$ are approximately twice as large. At $V>19 \mathrm{mag}$, the accuracy of photometry in the ultraviolet, especially for heavily reddened stars, is too low for reliable classification of stars earlier than K. For most of these stars the $U-V$ and $P-V$ colour indices are not given. Colour indices with $\sigma=0.05-0.10 \mathrm{mag}$ are labelled with colons. The stars found to be binaries or having asymmetric images were not classified in luminosity classes, and in the column of spectral types these stars are designated with double asterisks.

To verify the results of photometric classification, one of us (U.M.) obtained the spectra of 18 stars of the area down to $V=12 \mathrm{mag}$ with the $1.22 \mathrm{~m}$ telescope at the Asiago Observatory, Italy, equipped with a B\&C spectrograph and a 600 lines/mm grating providing a dispersion of $1.17 \AA /$ pix and a resolving power of $F W H M(\mathrm{PSF})=2.1$ pix. The recorded spectra cover 3487-5885 A. Spectral classification of these stars, listed in Table 3, was accomplished with the computer program MKCLASS designed to classify stellar spectra on the MK system provided by Gray \& Corbally (2014). Table 3 shows that in most cases the differences between the spectroscopic and photometric spectral types do not exceed one to two spectral subclasses and one luminosity class. This gives us additional confidence in two-dimensional spectral types determined from Vilnius photometry.
As an independent check of the classification of the Asiago spectra by means of the Gray \& Corbally (2014) code, we independently classified eight of them through a careful visual comparison of their calibrated spectra against the Yamashita et al. (1977) atlas of the representative stellar spectra in the MK system. For these stars (Nos. 8, 76, 279, 420, 565, 580, 668, and 683), differences between the spectral classification from photometry and from the Gray \& Corbally (2014) code were the largest. The results, listed in Table 3, are in strong support of the MKCLASS code.

\section{Interstellar extinctions and distances based on Vilnius photometry}

For 584 stars from Paper I and for 447 stars from Table 2 with reliable spectral and luminosity classes, colour excesses $E_{Y-V}$, interstellar extinctions $A_{V}$, and distances $d$ (in pc) were calculated with the following equations:

$$
\begin{aligned}
E_{Y-V} & =(Y-V)_{\mathrm{obs}}-(Y-V)_{0}, \\
A_{V} & =4.16 E_{Y-V}, \\
\log d & =0.2\left(V-M_{V}+5-A_{V}\right),
\end{aligned}
$$

where $V$ and $Y-V$ are the observed magnitudes and colour indices. The intrinsic colour indices $(Y-V)_{0}$ and absolute magnitudes $M_{V}$ for a given spectral type are from Straižys (1992). The errors of colour excesses, extinctions, and distances are described in Straižys et al. (2015), hereafter Paper II. Typical errors of the extinction and distance are $\sigma\left(A_{V}\right) / A_{V}= \pm 0.06$, and $\sigma(d) / d= \pm 0.10$.

In Eq. (2) the coefficient corresponds to the normal interstellar extinction law. The normality of the law was verified in the following way: (1) $123 \mathrm{~B}$ and A stars in the area were selected; (2) for these stars, $E_{B-V}=1.32 E_{Y-V}$ values were calculated; (3) the $E_{V-J}, E_{V-H}$, and $E_{V-K_{\mathrm{S}}}$ values were calculated taking the intrinsic colours from Straižys (1992); (4) the $R_{V}$ values were calculated from the ratios $E_{V-\lambda} / E_{B-V}$ with the equations from Fitzpatrick (1999), Fitzpatrick \& Massa (2009) and Larson (2014). The resulting $R_{V}$ values are between 2.92 and 3.12, i.e. close to the normal value 3.1 .

The plot $A_{V}$ vs. $d$ for stars in the whole $1.5 \mathrm{deg}^{2}$ area up to $500 \mathrm{pc}$ is shown in Fig. 2. The stars measured from the Moletai telescope exposures are shown as dots, and those measured from the VATT exposures are shown as open circles. The stars with spectroscopic MK types (Table 3 ) are plotted as crosses. The red vertical line designates the accepted cloud distance as discussed in the next section.

The $A_{V}$ vs. $d$ plot for 584 stars in the same area up to $5.5 \mathrm{kpc}$ is shown in Fig. 3. The two broken curves show the limiting magnitude effect for stars of spectral types $\mathrm{A} 0 \mathrm{~V}$ at $V=$ 15 and 16 mag. Most stars are located below the last curve. Above it, at large distances, we find only a few stars of spectral class $\mathrm{B}$ and $\mathrm{M}$ giants, which have absolute magnitudes brighter than A0 V. Figure 4 shows the $A_{V}$ vs. $d$ plot for 416 stars up to $5.5 \mathrm{kpc}$ in the three areas observed with the VATT telescope down to fainter apparent magnitudes.

\section{Distance to the TGU H994 P1 cloud}

The determination of distances to dust clouds located close to the Sun is difficult because the number of stars in the foreground and immediately behind the cloud is usually low, and most of them are $\mathrm{K}$ and $\mathrm{M}$ dwarfs. The beginning of the cloud should 
A\&A 585, A31 (2016)

Table 2. The first ten stars of the data catalogue in the three VATT areas measured in the Vilnius seven-colour system.

\begin{tabular}{|c|c|c|c|c|c|c|c|c|c|c|}
\hline No. & $\begin{array}{l}\text { RA (J2000) } \\
\text { h m s }\end{array}$ & $\begin{array}{c}\operatorname{Dec}(\mathrm{J} 2000) \\
0 \quad, \quad,\end{array}$ & $\begin{array}{l}V \\
\text { mag }\end{array}$ & $\begin{array}{l}U-V \\
\text { mag }\end{array}$ & $\begin{array}{l}P-V \\
\text { mag }\end{array}$ & $\begin{array}{l}X-V \\
\text { mag }\end{array}$ & $\begin{array}{l}Y-V \\
\text { mag }\end{array}$ & $\begin{array}{l}Z-V \\
\text { mag }\end{array}$ & $\begin{array}{l}V-S \\
\text { mag }\end{array}$ & $\begin{array}{l}\text { Phot. } \\
\text { sp. type }\end{array}$ \\
\hline 100 & 04:22:12.79 & $+54: 54: 13.9$ & 16.286 & 4.069 & 3.361 & 2.485 & 1188 & 0.463 & & $\mathrm{~g} 5 \mathrm{~V}$, md: \\
\hline 10 & 136 & & & & & & & & 0.998 & \\
\hline 1003 & $04: 22: 13.74$ & $+54: 49: 4$ & 18.029 & & 3. & 0 & & 0.406 & & f8 V \\
\hline 1004 & 04:22:14.00 & $+54: 53: 16.9$ & 18.4 & 4. & 3. & 2. & & 0.491 & 1.175 & g4 IV \\
\hline 1005 & $04: 22: 14.54$ & $+54: 52: 02.9$ & 16.544 & 4. & 3.066 & 2.0 & & 0.3 & 0.821 & a6 V \\
\hline 1006 & $04: 22: 14.56$ & $+54: 51: 39.1$ & 19.301 & & & 2.716 & 1.386 & 0.621 & 1.085 & g2 V \\
\hline 1007 & 04:22:14.77 & $+54: 47: 09.0$ & 19.483 & & 3.298 & 2.480 & 1.232 & 0.496 & & $\mathrm{~g} 2$ \\
\hline 1008 & $04: 22: 14.86$ & $+54: 46: 46.3$ & 17.831 & 4.070 & 3.159 & 2.214 & 1.099 & 0.437 & & $\mathrm{f} 2 \mathrm{~V}$ \\
\hline 1009 & 04:22:14.99 & $+54: 46: 29.5$ & 19.235 & & 3.151 & 2.239 & & 0.339 & & f5 V \\
\hline 1010 & 04:22:15.32 & $+54: 52: 00.2$ & 19.272 & & & 2.877 & 1.387 & 0.630 & 1.125 & $\mathrm{~g}$ \\
\hline
\end{tabular}

Notes. The stars are numbered starting from 1001 to avoid confusion with the catalogue of Paper I. The running numbers, coordinates, $V$ magnitudes, six colour indices, and photometric spectral types are given. The full catalogue of 855 stars is available at CDS.

Table 3. Stars classified from the Asiago spectra by the Gray \& Corbally (2014) code MKCLASS and by visual inspection (U.M.).

\begin{tabular}{|c|c|c|c|c|}
\hline \multirow[t]{2}{*}{ Star } & \multicolumn{2}{|c|}{ Asiago spectra } & \multirow{2}{*}{$\begin{array}{l}\text { Sp } \\
\text { KLASQ }\end{array}$} & \multirow{2}{*}{$\begin{array}{l}\text { Sp } \\
\text { COMPAR }\end{array}$} \\
\hline & MKCLASS & Visual & & \\
\hline 8 & G7 IV & G7 III-IV & $\mathrm{g} 8.5 \mathrm{~V}$ & g8 V \\
\hline 13 & $\mathrm{~A} 0 \mathrm{~V}$ & - & a $1.5 \mathrm{~V}$ & a1 V \\
\hline 39 & F7 V & - & f8 V & f8 V \\
\hline 65 & G9 III & - & g9.5 IV & g8 III-IV \\
\hline $76^{*}$ & B3/4 IV-V & B4/5 IV & b5 V & b5 Vp \\
\hline 162 & G0 IV-V & - & f8 IV & f8 IV-V \\
\hline 279 & G8 III-IV & G8/9 IV & g9.5 & k0 III-IV: \\
\hline 328 & K0.5 V & - & g9.5 V & $\mathrm{k} 0 \mathrm{~V}$ \\
\hline 341 & A1 IV-V & - & a0 IV & a1 V \\
\hline 396 & G9 V & - & $\mathrm{g} 8.5 \mathrm{~V}$ & g9 V \\
\hline 420 & B8 V & B7 V & $\mathrm{b} 8 \mathrm{~V}$ & b7 Vp \\
\hline 565 & B9 V & B9 & $\mathrm{a} 0 \mathrm{IV}$ & a2 V: \\
\hline 570 & F7 IV & - & f6/7 V & f7 V V \\
\hline 580 & M2.5 III & M2/3 III & $\mathrm{k}-\mathrm{m}$ & $\mathrm{k}-\mathrm{m}$ \\
\hline 601 & K4 V & - & $\mathrm{k} 4.2 \mathrm{~V}$ & k4 V \\
\hline 668 & F8 V & F8 V & f8 V & f6 V \\
\hline 683 & G4 III-IV & G5/6 IV & k0 IV & g8 III: \\
\hline HD & & & & \\
\hline 28097 & F7 V & F8 (HD) & - & - \\
\hline
\end{tabular}

Notes. For the comparison, photometric spectral types obtained with two different codes (KLASQ and COMPAR) are listed. ${ }^{(*)}$ No. 76 (HDE 232940) is a new Be-type star (Chojnowski et al. 2015) .

be signified by a sudden rise of the extinction at a certain distance. However, the apparent distance of the nearest reddened stars cannot be accepted as the cloud distance, since their distances are affected by the errors of the observed magnitudes and colours, photometric classification errors, cosmic dispersion of the intrinsic colours, variations in the shape of the interstellar extinction law; some stars can be unresolved binaries.

In our case (Fig. 2), the distribution of stars with distance shows a significant increase of the extinction starting from $\sim 110 \mathrm{pc}$. At about $200 \mathrm{pc}$ the extinction values close to $\sim 5$ mag are being reached. Trying to understand where the cloud is located, we must analyse the possible distance errors for each of the scarce reddened stars. The largest source of distance errors are luminosity classes determined by photometric classification. In different ranges of spectral classes, these errors are somewhat different, but their typical value is close to $3 \sigma= \pm 0.5 \mathrm{mag}$. For K- and M-type dwarfs the photometric luminosity class is usually correct, but in the HR diagram they

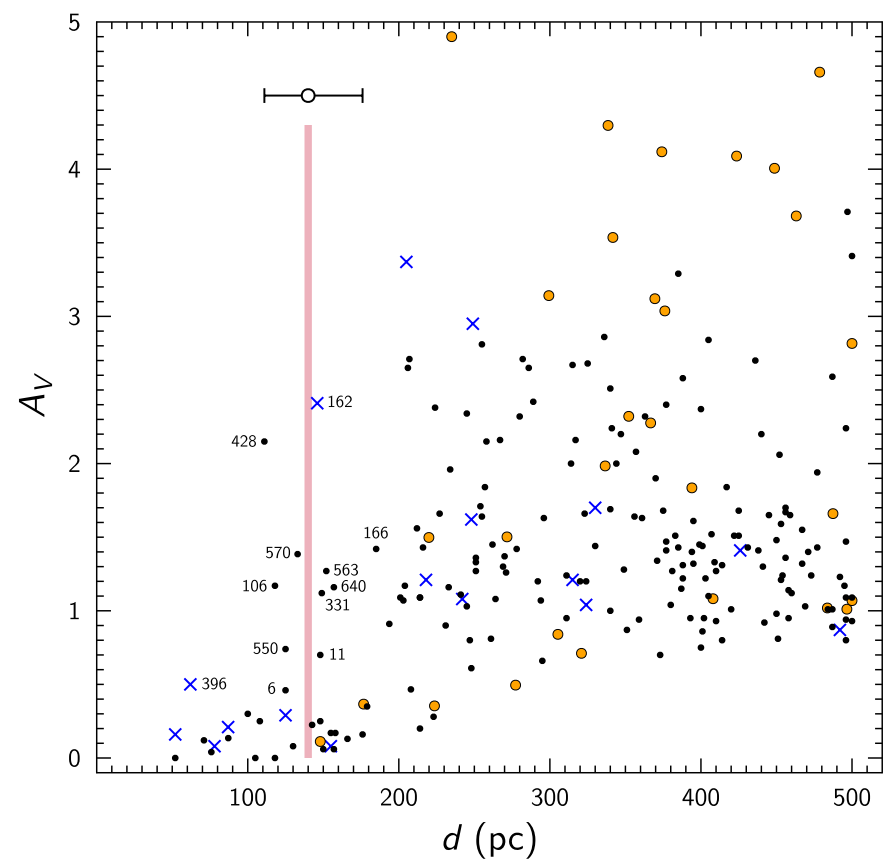

Fig. 2. Dependence of the extinction on distance for the investigated area up to $d=500 \mathrm{pc}$. The stars, measured in the Moletai telescope exposures, are plotted as dots, and the stars, measured in the VATT exposures, are plotted as circles. Crosses are the stars for which MK spectral classes were determined from their Asiago spectra (Table 3). The nearest reddened stars are labelled with their numbers in the Paper I catalogue. The red vertical line designates the accepted position of the dust cloud TGU H994 P1 at 140 pc (see the text). The $3 \sigma$ error bar for this distance is shown.

exhibit considerable intrinsic dispersion of their $M_{V}$ values at a fixed intrinsic colour or spectral class; see Perryman et al. (1995, 1997). An absolute magnitude error of $\pm 0.5 \mathrm{mag}$ leads to the minimum and maximum apparent distances $0.79 d$ and $1.26 d$. As result of these distance errors, stars move from the distance of the cloud to both sides, but they are easy to identify in the direction of negative distance errors (i.e. reddened stars moved to foreground) since in the direction of positive distance errors they become mixed with reddened background stars (except at $A_{V}$ close to zero, see below).

At this point, we evaluate the distance to the cloud from the apparent positions of the reddened foreground stars. Since there is no information about their binarity, the stars are considered to 
be moved from the cloud distance mainly by the errors of their absolute magnitudes. Unfortunately, the close reddened stars in our sample are very scarce.

The reddened star with $A_{V}>1 \mathrm{mag}$ that is nearest to us is No. $428\left(V=13.47, \mathrm{k} 1 \mathrm{~V}, A_{V}=2.14 \mathrm{mag}, d=111 \mathrm{pc}\right)$. This star is located only about $2^{\prime}$ north of the cloud LDN 1400 edge. If this star has been moved to this apparent distance by the $3 \sigma$ error, its real distance should be $111 \times 1.26=140 \mathrm{pc}$ (all distances in this paragraph are rounded to $1 \mathrm{pc}$ ). If star No. 428 is a binary or has been shifted shortwards by a larger distance error, the calculated cloud distance should be larger. If the star has been moved by a smaller distance error, its real distance, i.e. beginning of the cloud, should be smaller.

Another place in the $A_{V}$ vs. $d$ diagram is related to the unreddened or little reddened stars apparently located at larger distances than the cloud. If these stars have been moved to their apparent distances due to positive errors, then the real distance of the cloud should be smaller by a factor of 0.79 . In Fig. 2, the stars with small extinction $\left(A_{V}<0.2 \mathrm{mag}\right)$ extend to the apparent distance $180 \mathrm{pc}$, and this allows us to estimate the real cloud distance at $180 \times 0.79=142 \mathrm{pc}$. This value is in agreement with the distance given by the foreground star No. 428 .

If these considerations are real, the cloud distance is at $140 \mathrm{pc}$ with $3 \sigma$ errors $-31 \mathrm{pc}$ and $+36 \mathrm{pc}$ shown by the error bar in Fig. 2. Within this box, ten reddened stars with $A_{V}$ between 0.5 and 2.3 mag are present. On the other hand, we are not sure that the entire investigated area is covered by a single cloud located at a fixed distance. The cloud complex can have considerable depth along the sightview, and in this case our results should correspond to different dust condensations. We return to this possibility in Sect. 6 .

The star No. 396 ( $\left.V=10.15, \mathrm{G} 9 \mathrm{~V}, A_{V}=0.50, d=62 \mathrm{pc}\right)$, classified both from the Asiago spectra and from Vilnius photometry, also exhibits unusually large reddening for its distance. We suspect this may be caused by an unseen red dwarf companion.

Figures 3 and 4, which show the extinction vs. distance relations up to $5.5 \mathrm{kpc}$ for the Moletai and VATT telescopes, respectively, allow us to estimate the extinction run at large distances. The Molètai results demonstrate a strong limiting magnitude effect at $V=16 \mathrm{mag}$. Therefore, the stars with large extinction values (up to $\sim 3.7 \mathrm{mag}$ ) are only seen at distances closer than $2 \mathrm{kpc}$. In the VATT results, the limiting magnitude is close to $V=19 \mathrm{mag}$, and that is where we see the stars with $A_{V}$ up to 5 mag and at larger distances. Most of the heavily reddened stars are located close to the dark cloud LDN 1400 (VATT areas B and C). In Fig. 4, the absence of stars with $A_{V}$ lower than 2 mag can be explained by the fact that the VATT areas are located in directions with larger obscuration.

\section{The extinction based on 2MASS and WISE photometry of red clump giants}

RCGs offer an additional possibility to investigate the interstellar extinction in the area. These stars have a low dispersion of their intrinsic colours and absolute magnitudes (Perryman et al. 1995, 1997; Alves 2000; Grocholski \& Sarajedini 2002; Van Helshoecht \& Groenewegen 2007; Groenewegen 2008), and this allows us to use them as standard candles. In this section, we briefly describe their photometric identification with the 2MASS $J, H, K_{\mathrm{s}}$ (Skrutskie et al. 2006) and the WISE W2 (Wright et al. 2010) near-infrared systems.

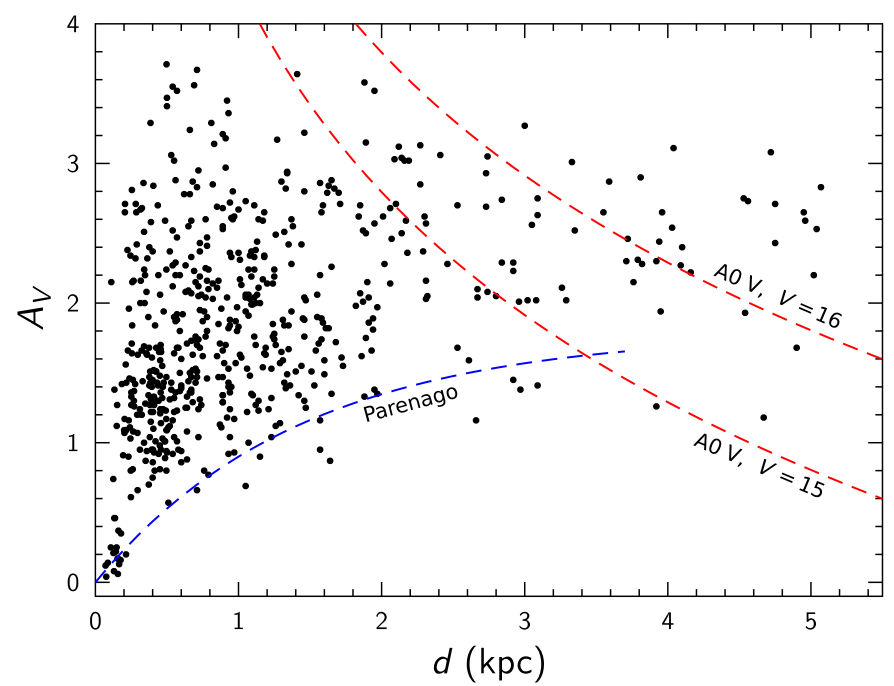

Fig. 3. Dependence of the extinction on distance for the investigated area up to $5.5 \mathrm{kpc}$ for 584 stars with two-dimensional classification, measured in the Moletai telescope exposures. The two broken curves show the limiting magnitude effect for $\mathrm{A} 0 \mathrm{~V}$ at $V=15$ and 16 mag. The exponential extinction formula of Parenago (1945) for the Galactic latitude $b=4^{\circ}, A_{V}=1.25 \mathrm{mag} / \mathrm{kpc}$ and the scale height $100 \mathrm{pc}$ is shown.

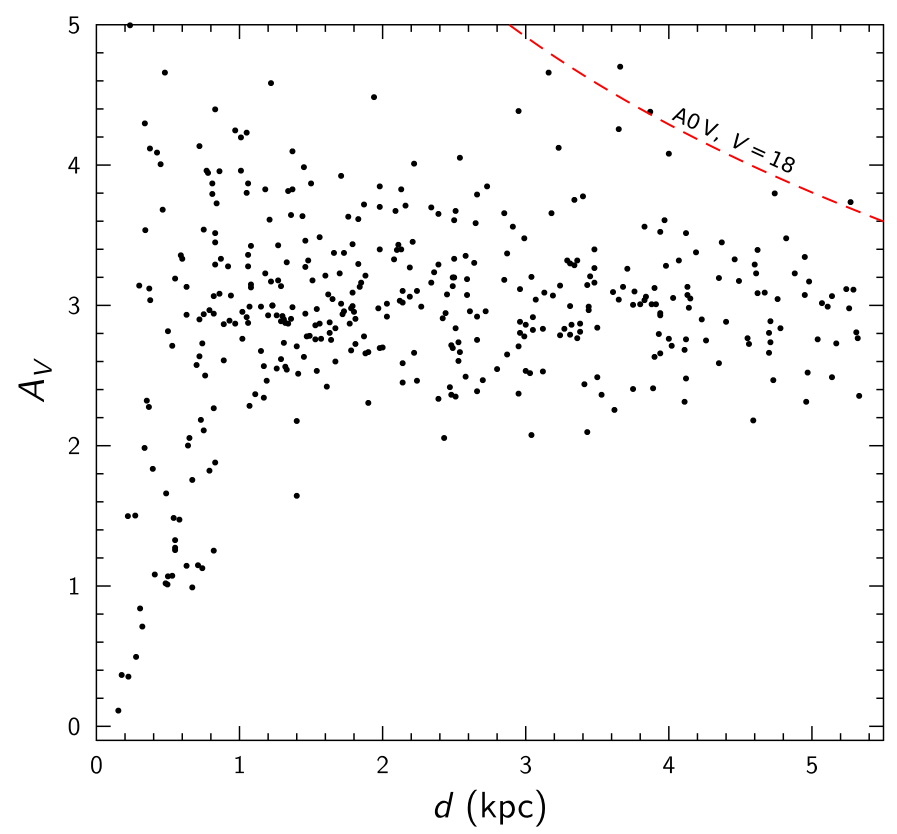

Fig. 4. Dependence of the extinction on distance in the three $12^{\prime} \times$ $12^{\prime}$ areas for 416 stars with two-dimensional classification, measured in the VATT telescope exposures. The red broken curve designates the limiting magnitude effect for A0 V stars at $V=18 \mathrm{mag}$.

\section{1. $\mathrm{JHK}_{\mathrm{s}}$ photometry}

Figure 5 shows the $J-H$ vs. $H-K_{\mathrm{s}}$ diagram plotted for 3230 stars in the investigated TGU H994 P1 area $\left(1.5 \mathrm{deg}^{2}\right)$ with the magnitude errors $\leq 0.03 \mathrm{mag}$. The intrinsic sequences of luminosity classes V and III, and the intrinsic position of RCGs are shown (Straižys \& Lazauskaite 2009). RCGs form a reddening belt which has the width $H-K_{\mathrm{s}} \approx 0.1 \mathrm{mag}$ and a slope of $E_{J-H} / E_{H-K_{\mathrm{S}}} \approx 2.0$ (Straižys \& Laugalys 2009). This belt covers the sequence of normal RGB stars of spectral classes G5 to M5 as well as unreddened or little reddened dwarfs of spectral 


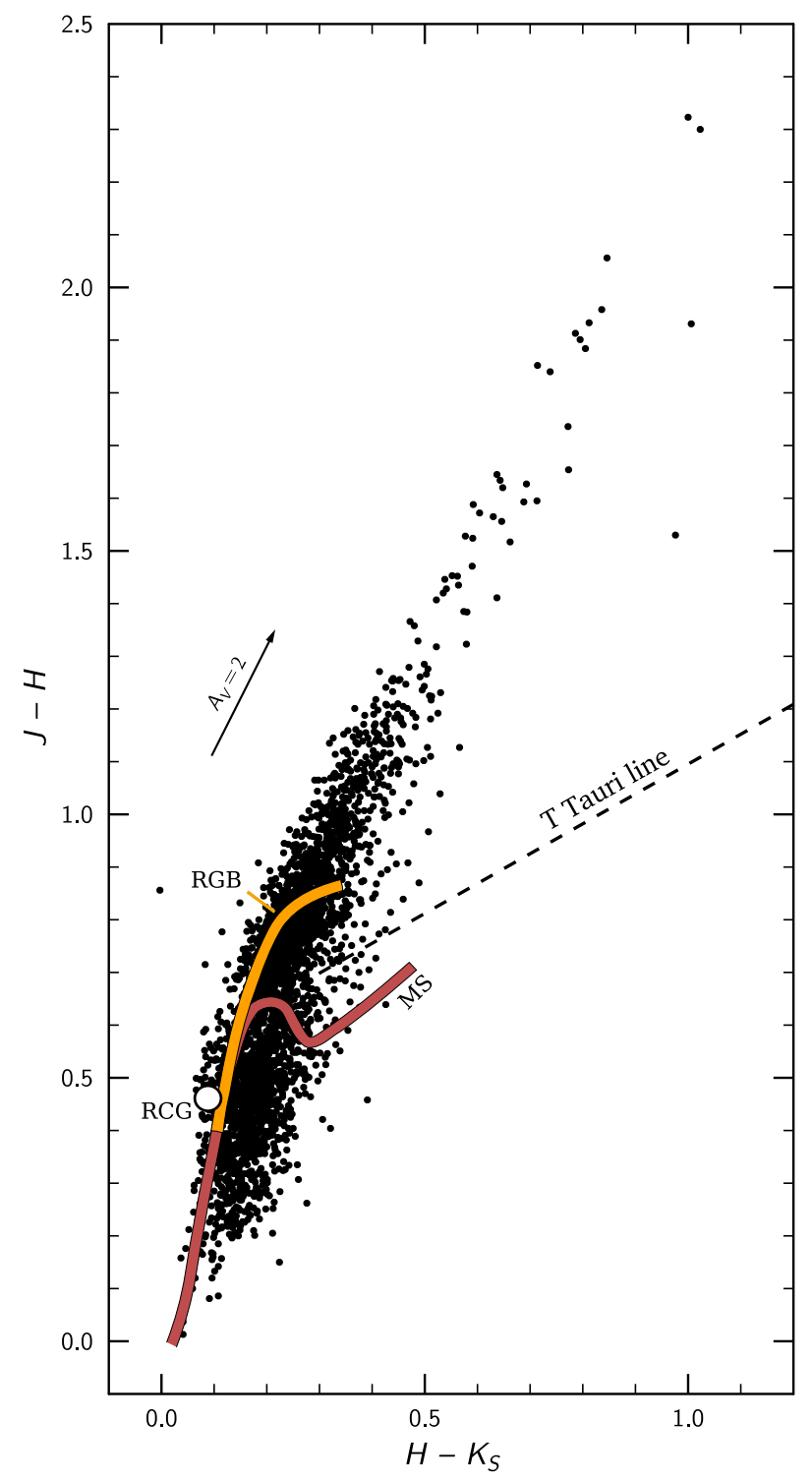

Fig. 5. $J-H$ vs. $H-K_{\mathrm{s}}$ diagram for 3230 stars in investigated TGU H994 P1 area. Only stars with the magnitude errors $\leq 0.03$ mag are plotted. The main sequence (MS, brown belt), the red giant branch (RGB, orange belt), the intrinsic locus of red clump giants (RCG, red circle), the intrinsic line of $\mathrm{T}$ Tauri stars (broken line) and the reddening line for $A_{V}=2$ mag are shown.

classes K and M0-M3. Thus, the 2MASS system alone does not allow us to separate RCGs from other types of stars.

The equation of the reddening line of RCGs,

$$
J-H=0.278+2.035\left(H-K_{\mathrm{s}}\right)
$$

was determined drawing it through the fixed intrinsic position of RCGs at $J-H=0.46, H-K_{\mathrm{s}}=0.09$ and 24 stars on the RCG belt for which $J-H>1.5$. At lower values of $J-H$, the reddening line is hidden within a crowding of stars of different temperatures, luminosities, and reddenings; see Fig. 5.

We also applied another, more reliable method to obtain the equation of the reddening line applying RCGs identified in the APOGEE high-resolution, near-infrared spectroscopic survey (Bovy et al. 2014). In the vicinity of the investigated area, we used 314 RCGs located in the four $3^{\circ}$ diameter circular areas listed in Table 4. One of these APOGEE areas partly overlaps a part of our area. We only took the magnitudes with the
Table 4. Four APOGEE areas in the vicinity of the investigated area from which RCGs were taken to determine equations of interstellar reddening lines in different near-infrared two-colour diagrams.

\begin{tabular}{lcccc}
\hline \hline $\begin{array}{l}\text { Name } \\
\text { of area }\end{array}$ & RA2000 & Dec2000 & $\begin{array}{c}\text { Number } \\
\text { of RCGs }\end{array}$ & $\begin{array}{c}\text { Range of } r \\
\text { mag }\end{array}$ \\
\hline GL490a & $03: 22$ & $+57: 00$ & 66 & $11.2-18.5$ \\
GL490b & $03: 42$ & $+60: 15$ & 65 & $12.6-16.4$ \\
NGC 1496 & $04: 04$ & $+52: 30$ & 62 & $11.7-17.2$ \\
TGU H944 & $04: 22$ & $+55: 15$ & 121 & $11.7-17.5$ \\
\hline
\end{tabular}

Notes. We only use the stars, for which magnitude errors are $\leq 0.03$. The areas are named according to the nearest Simbad object.

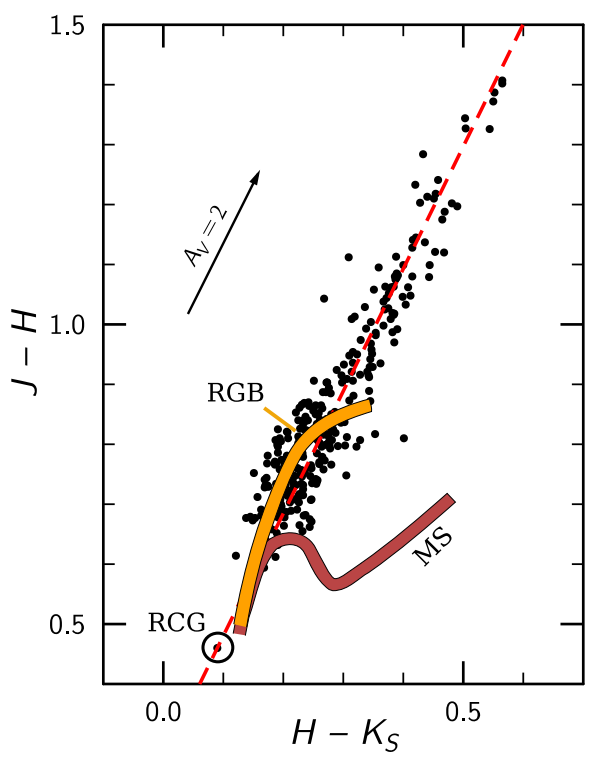

Fig. 6. $J-H$ vs. $H-K_{\mathrm{s}}$ diagram for the 314 RCGs in four areas of the APOGEE survey listed in Table 4 . Only the stars with the magnitude errors $\leq 0.03$ mag are plotted. The designation of sequences and other symbols is the same as in Fig. 5.

errors $\leq 0.03$ mag and without saturation. The $J-H$ vs. $H-K_{\mathrm{s}}$ diagram for these RCGs is shown in Fig. 6. The equation of the reddening line, again drawn through the fixed intrinsic position of RCGs, is

$J-H=0.279+2.031\left(H-K_{\mathrm{s}}\right)$,

which is very close to that obtained for the reddened RCGs mixed with the core H-burning RGB stars; see Eq. (4).

Next, we isolated 1667 stars that lie within the reddening belt of RCG and RGB stars limited by the following equations:

$J-H \leq 0.38+2.031\left(H-K_{\mathrm{s}}\right)$,

$J-H \geq 0.18+2.031\left(H-K_{\mathrm{s}}\right)$,

$J-H \geq 0.46$,

i.e. the belt width is $\Delta\left(H-K_{\mathrm{s}}\right)= \pm 0.10 \mathrm{mag}$, and it starts at the intrinsic position of RCGs (the circle in Fig. 5). In this belt, most RCGs should be present, but the $J-H$ vs. $H-K_{\mathrm{s}}$ diagram is not sufficient for their identification. To make this possible, we need to deredden all RCG+RGB stars lying within this belt.

\subsection{WISE photometry}

For dereddening of RCG and RGB stars in Paper II, the $H_{-}$ [4.5] vs. $J-[4.5]$ diagram has been used. Here [4.5] is the magnitude at $4.5 \mu \mathrm{m}$ of the Spitzer IRAC system. This diagram 
was proposed by Majewski et al. (2011) for the determination of colour excesses and extinctions of F-G-K stars since the intrinsic colour indices $H-[4.5]$ for most of them are close to zero. Since the Spitzer measurements in our area are absent, we changed [4.5] to the magnitude W2 of the WISE system with the mean wavelength at $4.6 \mu \mathrm{m}$ (Wright et al. 2010; Jarrett et al. 2011). To decrease contamination of the sample by dwarfs, we rejected the stars with $J-H<0.46$, the intrinsic colour of RCGs. The $H-W 2$ vs. $J-W 2$ diagram for 1320 stars, located in the $J-H$ vs. $H-K_{\mathrm{s}}$ diagram within the RCG+RGB reddening belt, is shown in Fig. 7. The W2 magnitudes are taken from the AllWISE Data Release (Cutri et al. 2012), and their errors are limited to $\pm 0.03 \mathrm{mag}$, as was the case with the magnitudes $J, H$, and $K_{\mathrm{s}}$.

For the determination of the equation of the reddening line of RCGs in the diagram $H-W 2$ vs. $J-W 2$, we applied the same set of RCGs from four nearby areas of the APOGEE survey, as in the case of Fig. 6. Figure 8 shows that RCGs form a quite tight reddening line with a slope close to $E_{H-W 2} / E_{J-W 2}=0.51$. The intrinsic point of RCGs in this diagram was determined applying two old open clusters, M67 and NGC 2506, which contain compact concentrations of RCGs and have small reddening ( $E_{B-V}=0.06$ and 0.05 , respectively). We selected four RCGs in M67 and nine RCGs in NGC 2506, free of close neighbours and with the errors of all magnitudes $\leq 0.03 \mathrm{mag}$. These 13 RCGs after dereddening are plotted in Fig. 8 (the lower compact group of dots), where they define the intrinsic position of RCGs at $H-W 2=0.07$ and $J-W 2=0.56$. The equation of the reddening line drawn through the APOGEE RCGs and the fixed intrinsic position is:

$H-W 2=-0.217+0.512(J-W 2)$.

\subsection{Identification of RCGs}

We isolated RCGs in the $H-W 2$ vs. $J-W 2$ diagram of the investigated area (Fig. 7) with the following equations:

$H-W 2 \leq-0.17+0.51(J-W 2)$
$H-W 2 \geq-0.27+0.51(J-W 2)$

i.e. the width of the belt $\Delta(H-W 2)=0.1 \mathrm{mag}$ has been taken, which corresponds to the scatter of the APOGEE RCGs (Fig. 8).

This list of RCGs, however, includes a considerable amount of unreddened and reddened dwarfs of spectral classes $\mathrm{G}-\mathrm{K}-\mathrm{M}$, and subgiants of spectral classes $\mathrm{G}-\mathrm{K}$. For their elimination, we applied the magnitude-colour diagram $K_{\mathrm{s}}$ vs. $H-K_{\mathrm{s}}$. In this diagram, late-type stars of luminosities V and IV form a clump of stars at the lower left corner with the vertical extension upwards along the $H-K_{\mathrm{s}}=0.1$ line; see Fig. 5 in Paper II. These stars were eliminated with the equations

$H-K_{\mathrm{s}}<0.15$

and

$K_{\mathrm{s}}>11+5\left(H-K_{\mathrm{s}}\right)$

After application of the described procedures, the resulting list contains 837 suspected RCG stars. Undoubtedly, this list is contaminated by a certain amount of normal $\mathrm{G}-\mathrm{K}$ giants and subgiants of RGB as well as K-M dwarfs. The percentage of this contamination and its possible consequences are estimated in the next section.

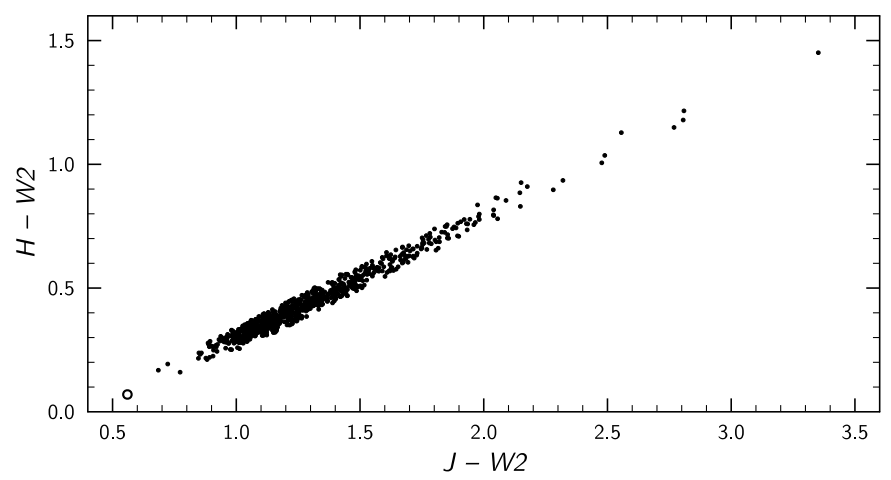

Fig. 7. $H-W 2$ vs. $J-W 2$ diagram for the investigated H994 $\mathrm{P} 1$ area. The stars from the RCG+RGB belt in the $J-H$ vs. $H-K_{\mathrm{s}}$ diagram with the 2MASS and WISE magnitude errors $\leq 0.03$ mag are plotted.

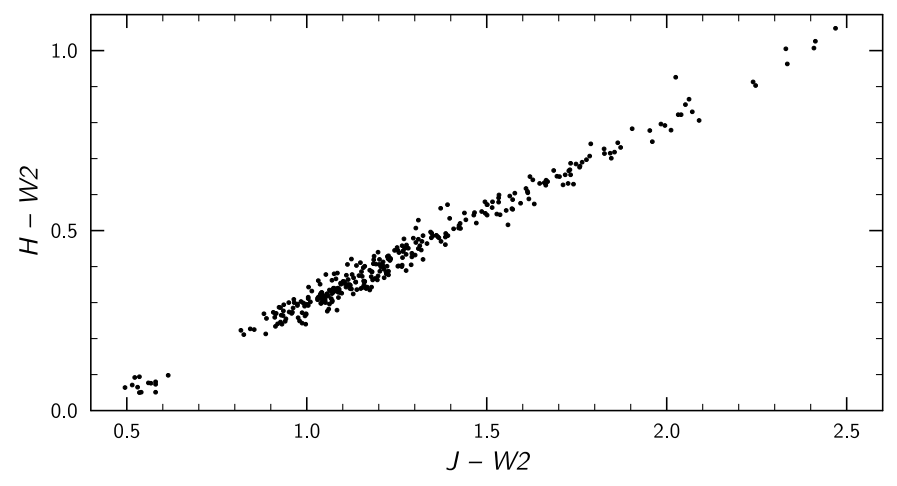

Fig. 8. $H-W 2$ vs. $J-W 2$ diagram for the 314 RCGs in four areas of the APOGEE survey listed in Table 4 . We plot only the stars with the magnitude errors $\leq 0.03 \mathrm{mag}$. The group of 13 dots at $H-W 2=0.07$, $J-W 2=0.56$ are dereddened RCGs of clusters M67 and NGC 2506, defining the intrinsic position of RCGs.

Colour excesses of the selected RCGs were determined as

$$
\begin{aligned}
E_{H-W 2} & =(H-W 2)_{\mathrm{obs}}-0.07, \\
E_{J-H} & =0.96 E_{H-W 2}, \\
E_{H-K_{\mathrm{s}}} & =0.47 E_{H-W 2} .
\end{aligned}
$$

The last two equations were derived from the corresponding ratios for the Spitzer [4.5] mag (Paper II), and the equation

$E_{H-W 2}=1.035 E_{H-[4.5]}$,

which we derived from the normal interstellar extinction law presented by McClure (2009).

In Fig. 9 dereddened RCGs are plotted in the $(J-H)_{0}$ vs. $\left(H-K_{\mathrm{s}}\right)_{0}$ diagram. For control, in Fig. 10 we plot the same diagram for 314 APOGEE RCGs from the four areas listed in Table 4 and 13 RCGs from open clusters M67 and NGC 2506, all dereddened with the same method. In both figures, RCGs cover very similar areas. In Fig. 9 the RCG area has a form of a parallelogram with the sides defined by the RCG belts: the right and left sides are limited by Eqs. (6) and (7), and the lower and upper sides by $(J-H)_{0}=0.40$ and 0.60 , which follow from Eqs. (10), (11), (14), and (15). In both figures the centre of the largest concentration of RCGs corresponds to $(J-H)_{0}=0.48$ and $\left(H-K_{\mathrm{s}}\right)_{0}=0.10$. Below we accept these values as intrinsic colours of RCGs. They are larger than the intrinsic colours of RCGs estimated by Straižys \& Lazauskaite (2009) just by 0.02 and $0.01 \mathrm{mag}$.

Trying to decrease the contamination, we excluded all stars lying outside the rectangle centred on the intrinsic position of 


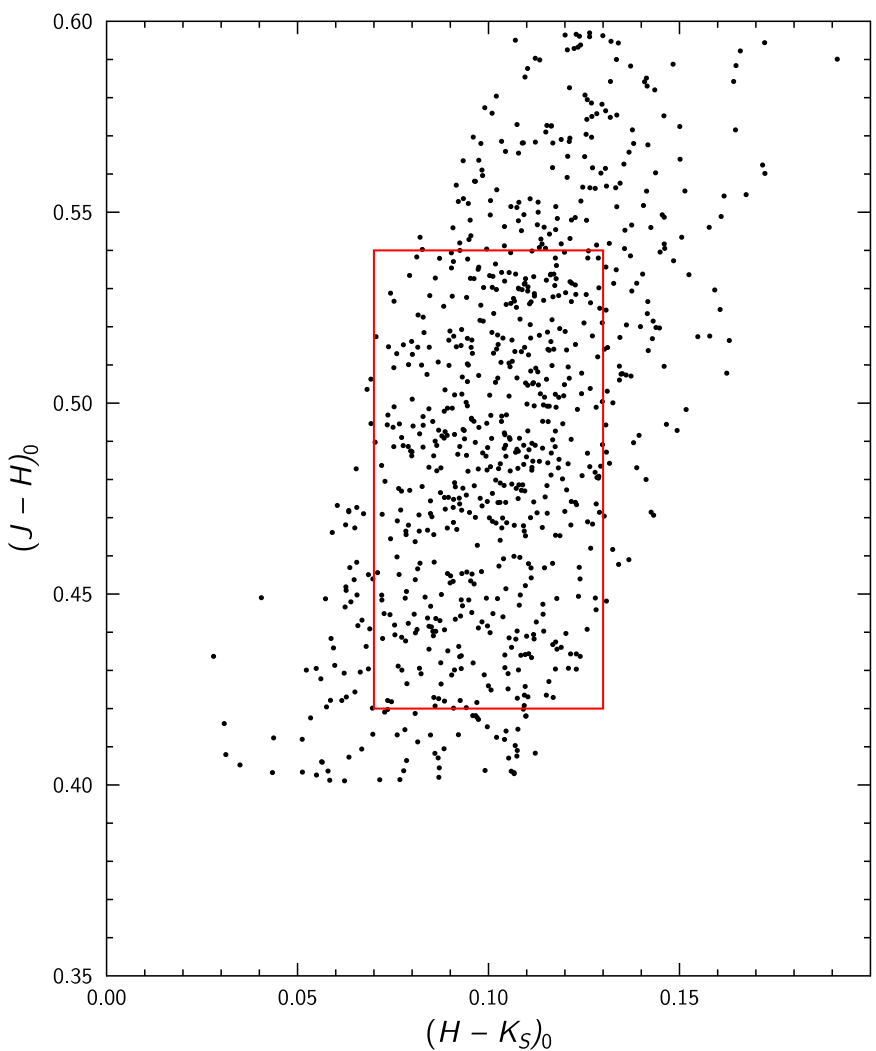

Fig. 9. Intrinsic $(J-H)_{0}$ vs. $\left(H-K_{\mathrm{s}}\right)_{0}$ diagram for 837 stars in the investigated TGU H994 P1 area, the majority of which should be RCGs. Only the stars located inside the red rectangle were selected to investigate the extinction.

RCGs with side lengths of 0.12 mag in $J-H$ and 0.06 mag in $H-K_{\mathrm{s}}$. Within this rectangle 504 stars remain.

\subsection{Interstellar extinction of RCGs}

Interstellar extinctions and distances of the selected RCGs were determined with the following equations:

$$
\begin{aligned}
A_{K_{\mathrm{s}}} & =0.67 E_{J-K_{\mathrm{s}}}=0.67\left[\left(J-K_{\mathrm{s}}\right)_{\mathrm{obs}}-\left(J-K_{\mathrm{s}}\right)_{0}\right], \\
\log d & =0.2\left(K_{\mathrm{s}}-M_{K_{\mathrm{s}}}+5-A_{K_{\mathrm{s}}}\right),
\end{aligned}
$$

where $\left(J-K_{\mathrm{s}}\right)_{0}=0.58$ and $M_{K_{\mathrm{s}}}=-1.6 \mathrm{mag}$. The relative errors of the extinction and distance are $\sigma\left(A_{K_{\mathrm{s}}}\right)= \pm 0.115 A_{K_{\mathrm{s}}}$ and $\sigma(d) / d= \pm 0.042$ (Paper II).

Figure 11 shows the $A_{K_{\mathrm{s}}}$ vs. $d$ plot for the selected RCGs in the investigated area. The right $y$-axis shows the extinctions transformed to the $A_{V}$ scale with the equation

$A_{V}=8.3 A_{K_{\mathrm{s}}}$,

which is valid for the normal interstellar extinction law.

\section{Discussion}

The distance to the cloud TGU H994 P1 estimated in Sect. 4 is based on the assumption that the reddened star No. 428 (k1 V) has appeared at $111 \mathrm{pc}$ owing to a negative $3 \sigma$ distance error. Then the true distance of this star should be at $140 \mathrm{pc}$, where the cloud causing its reddening is located. This result is also supported by a few stars with low extinction and distances up to $180 \mathrm{pc}$. If these stars are located in front of the cloud, i.e.

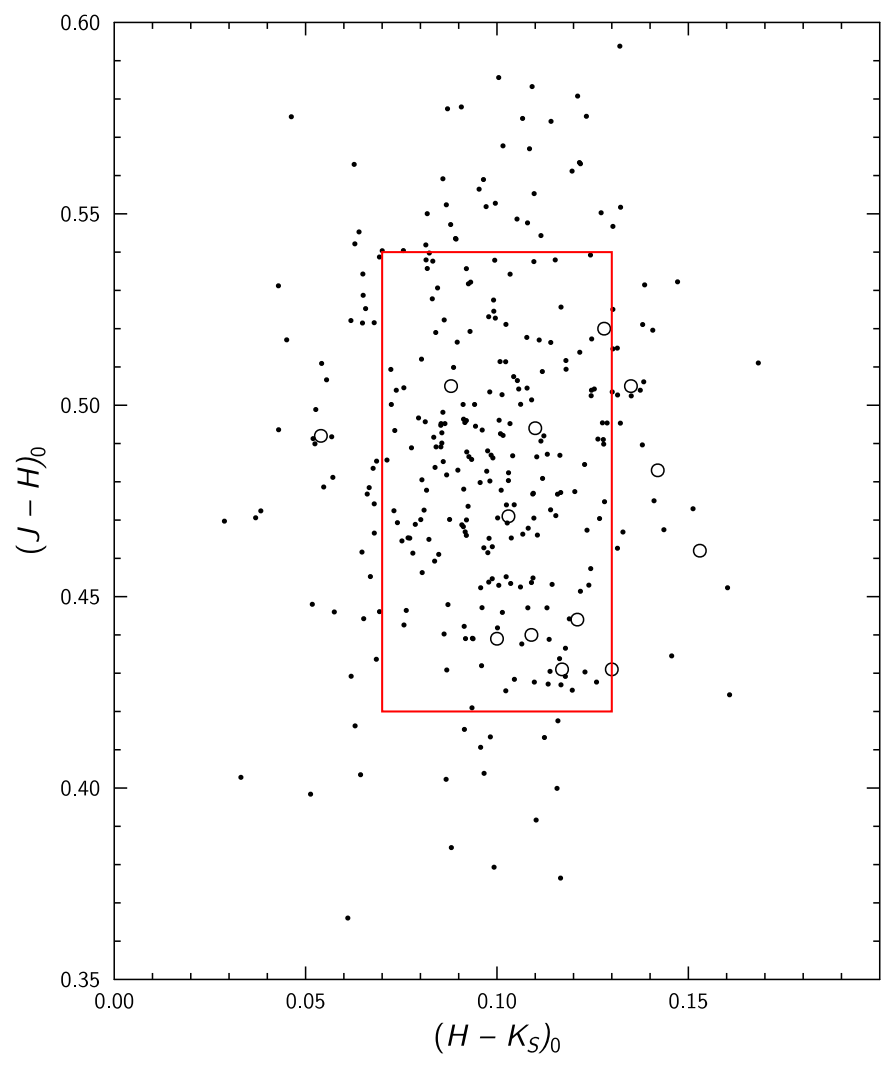

Fig. 10. Intrinsic $(J-H)_{0}$ vs. $\left(H-K_{\mathrm{s}}\right)_{0}$ diagram for 314 dereddened RCGs from the four APOGEE areas (Table 4, dots) and from the open clusters M67 and NGC 2506 (circles). The red rectangle is duplicated from Fig. 9.

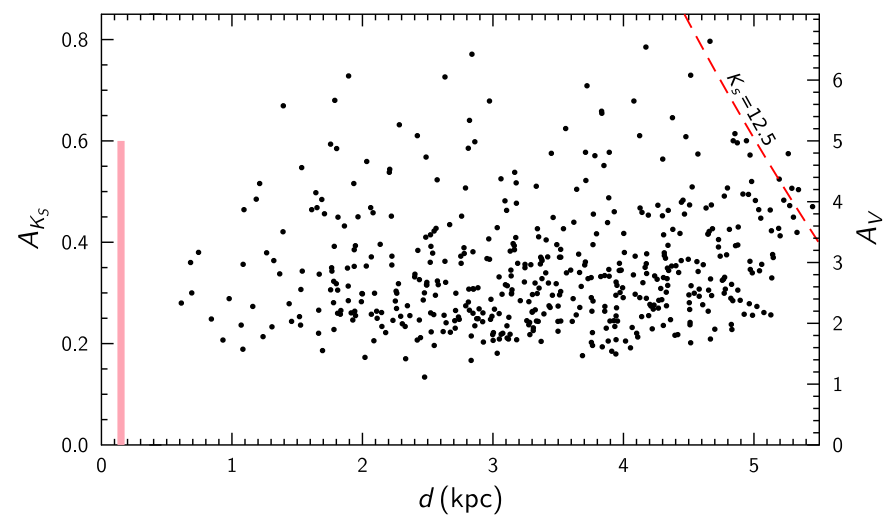

Fig. 11. Extinction $A_{K_{\mathrm{s}}}$ vs. distance in the investigated area for the RCGs identified combining the 2MASS and WISE magnitudes. The vertical line designates the accepted position of the cloud TGU H994 P1 at $140 \mathrm{pc}$. The red broken curve designates RCGs at the limiting magnitude $K_{\mathrm{s}}=12.5 \mathrm{mag}$.

at $140 \mathrm{pc}$, they could be shifted to their apparent positions because of positive $3 \sigma$ distance errors. A distance of $140 \mathrm{pc}$ is in agreement with the distance to the complex of the Taurus dark clouds located on the opposite side of the Milky Way (see references in Introduction). On the other hand, the Camelopardalis complex of dark clouds can be very deep along the sightline, as is the case with the Taurus complex, which has a depth of up to $50 \mathrm{pc}$ or more, determined from radio interferometry of young stellar objects (Loinard et al. 2011) and from interstellar line strengths (Welsh et al. 2010; Lallement et al. 2014) combined with the Hipparcos parallaxes. 


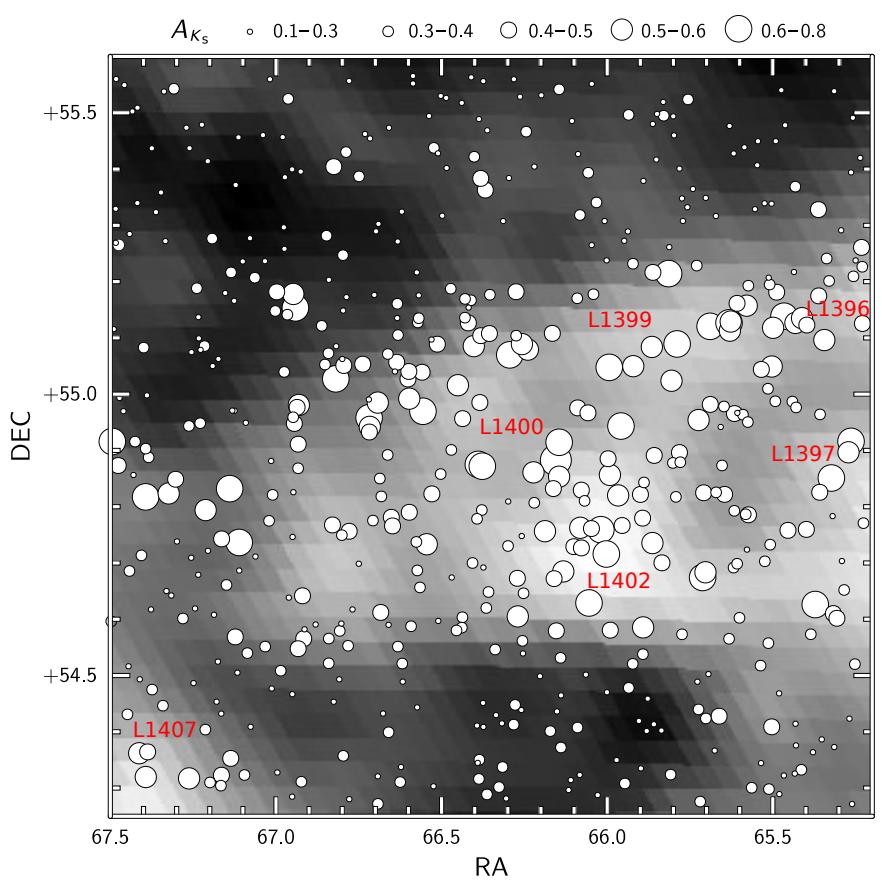

Fig. 12. Surface distribution of the extinction for RCGs. The five sizes of the circles correspond to different ranges of extinction. The five Lynds clouds are identified. The dust map from Schlegel et al. (1998) is shown in the background.

The investigated area is located at $b=\sim 4^{\circ}$, and this means that with increasing distance our sightline recedes from the Galactic plane: the height above the plane is $70 \mathrm{pc}$ at $1 \mathrm{kpc}$, $140 \mathrm{pc}$ at $2 \mathrm{kpc}, 280 \mathrm{pc}$ at $4 \mathrm{kpc}$, etc. The expected extinction rise due to the diffuse dust layer near the plane (the Parenago law, Fig. 3) at $d>1 \mathrm{kpc}$ should be rather low. Therefore, the extinction level at large distances must be defined mainly by the cloud with an additional small component caused by the diffuse dust layer. This is confirmed by the extinction versus distance plot in Fig. 4, where the results of deep classification of stars up to $\sim 19$ mag are shown. The minimum extinction in the most transparent directions remains at about 1.5-2 mag, and the maximum extinction in the directions where our sightline crosses peripheries of the dense clouds is between 4 and 5 mag. At the distances of the Perseus Arm and the Outer Arm, our sightline is about $100-140 \mathrm{pc}$ and $350 \mathrm{pc}$ above the plain. Thus, the influence of dust content in these two arms is not detectable.

Figure 11 shows that RCGs exhibit more or less the same minimum extinction level close to $A_{V}=1.5-2$ found for the other stars classified in the Vilnius system (Figs. 3 and 4). However, the maximum extinction is larger by about 1.5-2.5 mag, reaching about $6.5 \mathrm{mag}$ in the dark filaments and around them.

In Fig. 12 we plot a map of RCGs identified in the investigated area with different sizes of circles for different ranges of the extinction. The smallest circles are for $A_{K_{\mathrm{s}}}$ between 0.1 and $0.3 \mathrm{mag}$, and the largest circles for $A_{K_{\mathrm{s}}}$ higher than $0.6 \mathrm{mag}$. In the background the dust map from Schlegel et al. (1998) based on the IRAS and COBE/DIRBE $100 \mu \mathrm{m}$ emission observations is shown. It is evident that the largest circles concentrate on the darkest dust filaments, especially at LDN 1399 , LDN 1400, and LDN 1402. The calibration of the dust maps by Schlafly \& Finkbeiner (2011) in these clouds gives the values of $A_{V}$ between 4.5 and $4.9 \mathrm{mag}^{3}$ in a good agreement with the values given by RCGs.

It is important to estimate the contamination of the identified RCG sample by stars of other types. Probably, most latetype dwarfs have been excluded by the constraints applied to the $J-H$ vs. $H-K_{\mathrm{s}}, K_{\mathrm{s}}$ vs. $H-K_{\mathrm{s}}$, and $H-W 2$ vs. $J-W 2$ diagrams. However, the final list of RCGs can be contaminated by some amount of G8-K0 hydrogen-burning giants and subgiants of RGB. In our Paper II, we estimated about 50\% contamination. In the present investigation the contamination percent must be smaller since for the calibration of the method we applied a large sample of true RCG stars selected with the physical parameters of stars determined from high-dispersion spectra. Since the reddened APOGEE RCGs were taken from nearby areas, the slopes of the interstellar reddening lines in two-colour diagrams should be free of the Galactic longitude effect.

The list of selected RCGs contained 46 stars classified with Molètai photometry and 26 stars classified with VATT photometry. Among the Molètai stars, 13 have been classified as dwarfs or subgiants, and this constitutes $28 \%$ of common stars. Among the VATT stars, the contamination by dwarfs and subgiants is about $20 \%$. These wrong RCGs exhibit low values of the extinction at large distances; they were all excluded from the $A_{K_{\mathrm{s}}}$ vs. $d$ plot (Fig. 11). We have no possibility to estimate the percentage of contamination by RGB G8-K0 giants. Fortunately, both RCGs and RGB giants of the same spectral classes have similar intrinsic colours $(J-K)_{0}$ and absolute magnitudes $M_{K_{\mathrm{s}}}$ (see Paper II). Thus, their positions in the $A_{K_{\mathrm{s}}}$ vs. $d$ plot should be not very different.

The clouds in the investigated area do not show active star formation. Radio observations in molecular lines reveal a number of cool gas cores of the subparsec sizes, which might be prestellar clumps. However, stellar YSOs in the area are quite scarce. In Fig. 5, only a few stars are located in the region of possible T Tauri stars. Among them, the most reliable candidate is 2 MASS J04251408+5456524 at $J-H=1.53$ and $H-K_{\mathrm{s}}=0.97$. Two more possible YSOs, 2MASS J04241953+5453594 and J04262976+5450399, are revealed by the $K_{\mathrm{s}}-W 1$ vs. $W 1-W 2$ diagram. These three stars all are seen in the direction of LDN 1400. A few stars located in Fig. 5 above the intrinsic T Tauri line (from Meyer et al. 1997) with $J-H=0.8-1.1$ and $H-K_{\mathrm{s}}=0.4-0.6$ are candidates to be YSOs of class III (WTTS). These stars cannot be normal $\mathrm{M}$ dwarfs reddened by interstellar dust since in this case their distances calculated with Eq. (19) are unrealistically small (50$80 \mathrm{pc}$ ). Part of them can be binaries whose reddening is caused by a cooler secondary component.

\section{Conclusions}

We present the results of seven-colour photometry of 855 stars in the Vilnius photometric system down to $V \approx 20$ mag in the vicinity of the dark cloud TGU H994 P1 (Dobashi et al. 2005). For about $65 \%$ of these stars, we determine their spectral and luminosity classes, colour excesses, extinctions $A_{V}$, and distances. The new data supplement the catalogue of 727 stars in a larger area down to $V \approx 17 \mathrm{mag}$ published earlier (Čepas et al. 2013b). For control, MK types of the 18 brightest stars were determined from the spectrograms obtained at the Asiago Observatory. In addition, we develop a method to identify red clump giants, applying infrared photometry from 2MASS and WISE surveys.

The NASA/IPAC Extragalactic Database, NED. 
The method is calibrated with the use of four areas of the highdispersion spectral APOGEE survey, located in the vicinity of the investigated area. We used this method to identify 504 RCGs in the area down to $K_{\mathrm{s}}=12.5 \mathrm{mag}$.

The results of photometric classification of about 1000 stars are applied to investigate the interstellar extinction in the area, covering about $1.5 \mathrm{deg}^{2}$ and to estimate the distance to the dust cloud. At the distances $>1 \mathrm{kpc}$, this investigation is supported by the extinctions of 504 RCGs. We found the distance to the cloud TGU H994 P1, based on a few stars, classified by the Vilnius colour indices and spectroscopically, to be close to $140 \pm 11 \mathrm{pc}$. This result coincides with a distance of the Taurus dark clouds located on the opposite side of the Galactic equator. Alternative distances are also discussed. We do not exclude that the cloud complex in the investigated area has a considerable depth, such as the star-forming region in Taurus. Verification of distances to the reddened TGU H994 P1 stars will be soon possible with Gaia parallaxes.

The minimum extinction in the area of the order of 1.0-1.5 mag is well defined by the exponential Parenago law with $A_{V}=1.25 \mathrm{mag} / \mathrm{kpc}$. We observe maximum $A_{V}$ extinction up to about $6.5 \mathrm{mag}$ in the dark cloud filaments and their vicinities. At distances $>1 \mathrm{kpc}$, the mean extinction does not increase up to $5.5 \mathrm{kpc}$. This result is expected since our sightline crosses the Galaxy at 100-140 pc above the Perseus Arm and at $~ 350$ pc above the Outer Arm.

Acknowledgements. The use of the Simbad and SkyView databases is acknowledged. The project is partly supported by the Research Council of Lithuania, grant No. MIP-061/2013.

\section{References}

Alves, D. R. 2000, ApJ, 539, 732

Barnard, E. E. 1927, Vizier Online Data Catalogue: VII/220A

Benson, P. J., \& Myers, P. C. 1989, ApJS, 71, 89

Bovy, J., Nidever, D. L., Rix, H. W., et al. 2014, ApJ, 790, 127

Čepas, V., Zdanavičius, J., Zdanavičius, K., Straižys, V., \& Laugalys, V. 2013a, Baltic Astron., 22, 223

Čepas, V., Zdanavičius, J., Zdanavičius, K., Straižys, V., \& Laugalys, V. 2013b, Baltic Astron., 22, 243, Paper I

Chojnowski, S. D., Whelan, D. G., \& Wisniewski, J. P. 2015, AJ, 149, 7

Clark, F. O. 1991, ApJS, 75, 611

Cutri, R. M. et al. 2012, VizieR Online Data Catalog: II/311

Dame, T. M., Ungerechts, H., Cohen, R. S., et al. 1987, ApJ, 322, 706

Dame, T. M., Hartmann, D., \& Thaddeus, P. 2001, ApJ, 547, 792

de Zeeuw, P. T., Hoogerwerf, R., de Bruijne, J. H. J., Brown, A. G. A., \& Blaauw, A. 1999, AJ, 117, 354

Dickman, R. L. 1975, ApJ, 202, 50

Dieter, N. H. 1973, ApJ, 183, 449

Dobashi, K. 2011, PASJ, 63, S1

Dobashi, K., Uehara, H., Kandori, R., et al. 2005, PASJ, 57, S1

Dutra, C. M., \& Bica, E. 2002, A\&A, 383, 631

Fitzpatrick, E. L. 1999, PASP, 111, 63

Fitzpatrick, E. L., \& Massa, D. 2009, ApJ, 699, 1209

Gray, R. O., \& Corbally, C. J. 2014, AJ, 147, 80
Grocholski, A. J., \& Sarajedini, A. 2002, AJ, 123, 1603

Groenewegen, M. A. T. 2008, A\&A, 488, 935

Harjunpää, P., Kaas, A. A., Carlqvist, P., \& Gahm, G. F. 1999, A\&A, 349, 912

Jarrett, T. H., Cohen, M., Masci, F., et al. 2011, ApJ, 735, 112

Juvela, M., Ristorcelli, I., Pagani, L., et al. 2012, A\&A, 541, A12

Lallement, R., Welsh, B. Y., Vergely, J.-L., et al. 2003, A\&A, 411, 447

Lallement, R., Vergely, J.-L., Valette, B., et al. 2014, A\&A, 561, A91

Larson, K. A. 2014, PASP, 126, 27

Laugalys, V., Kazlauskas, A., Boyle, R. P., et al. 2004, Baltic Astron., 13, 1

Lee, C. W., \& Myers, P. C. 1999, ApJS, 123, 233

Lindblad, P. O. 1974, in Kinematics and Ages of Stars Near the Sun, ed. L. Perek, 381

Lindblad, P. O., Grape, K., Sandqvist, A., \& Schober, J. 1973, A\&A, 24, 309

Loinard, L., Mioduszewski, A. J., Torres, R. M., et al. 2011, in Rev. Mex. Astron. Astrofis. Conf. Ser., 40, 205

Lynds, B. T. 1962, ApJS, 7, 1

Majewski, S. R., Zasowski, G., \& Nidever, D. L. 2011, ApJ, 739, 25

McClure, M. 2009, ApJ, 693, L81

Meyer, M. R., Calvert, N., \& Hillenbrand, L. A. 1997, AJ, 114, 288

Milašius, K., Boyle, R. P., Vrba, F. J., et al. 2013, Baltic Astron., 22, 181

Minn, Y. K., \& Greenberg, J. M. 1973, A\&A, 22, 13

Myers, P. C., \& Benson, P. J. 1983, ApJ, 266, 309

Parenago, P. P. 1945, AZh, 22, 129

Park, Y., Lee, C. W., \& Myers, P. C. 2004, ApJS, 152, 81

Perryman, M. A. C., Lindegren, L., Kovalevsky, J., et al. 1995, A\&A, 304, 69

Perryman, M. A. C., Lindegren, L., Kovalevsky, J., et al. 1997, A\&A, 323, L49

Roeser, S., Demleitner, M., \& Schilbach, E. 2010, AJ, 139, 2440

Sandqvist, A., Tomboulides, H., \& Lindblad, P. O. 1988, A\&A, 205, 225

Schlafly, E. F., \& Finkbeiner, D. 2011, ApJ, 737, 103

Schlegel, D. J., Finkbeiner, D. P., \& Davis, M. 1998, ApJ, 500, 525

Sfeir, D. M., Lallement, R., Crifo, F., \& Welsh, B. Y. 1999, A\&A, 346, 785

Skrutskie, M. F., Cutri, R. M., Stiening, R., et al. 2006, AJ, 131, 1163

Snell, D. M. 1981, ApJS, 45, 121

Straižys, V. 1992, Multicolor Stellar Photometry (Tucson, Arizona: Pachart Publishing House)

http://www.itpa.1t/MulticolorStellarPhotometry/

Straižys, V., \& Laugalys, V. 2007, Baltic Astron., 16, 167

Straižys, V., \& Laugalys, V. 2008, in Handbook of Star Forming Regions 1, ed. B. Reipurth (ASP Monograph Publications), 294

Straižys, V., \& Laugalys, V. 2009, Baltic Astron., 18, 141

Straižys, V., \& Lazauskaitè, R. 2009, Baltic Astron., 18, 19

Straižys, V., Boyle, R. P., Janusz, R., et al. 2013, A\&A, 554, A3

Straižys, V., Vrba, F. J., Boyle, R. P., et al. 2015, AJ, 149, 161, Paper II

Taylor, D. K., Dickman, R. L., \& Scoville, N. Z. 1987, ApJ, 315, 104

Van Helshoecht, V., \& Groenewegen, M. A. T. 2007, A\&A, 463, 559

Vergely, J.-L., Ferrero, R. F., Siebert, A., \& Valette, B. 2001, A\&A, 366, 1016

Vergely, J.-L., Valette, B., Lallement, R., \& Raimond, S. 2010, A\&A, 518, A31

Welsh, B. Y., Craig, N., Vedder, P. W., \& Vallerga, J. V. 1994, ApJ, 437, 638

Welsh, B. Y., Lallement, R., Vergely, J.-L., \& Raimond, S. 2010, A\&A, 510, A54

Wright, E. L., Eisenhardt, P. R. M., \& Mainzer, A. K. 2010, AJ, 140, 1868

Wu, Y., Liu, T., Meng, F., et al. 2012, ApJ, 756, 76

Yamashita, Y., Nariai, K., \& Norimoto, Y. 1977, An Atlas of Representative Stellar Spectra (University of Tokyo Press)

Zdanavičius, J., \& Zdanavičius, K. 2002a, Baltic Astron., 11, 75

Zdanavičius, J., \& Zdanavičius, K. 2002b, Baltic Astron., 11, 441

Zdanavičius, J., \& Zdanavičius, K. 2005, Baltic Astron., 14, 1

Zdanavičius, J., Zdanavičius, K., \& Kazlauskas, A. 1996, Baltic Astron., 5, 563

Zdanavičius, J., Černis, K., Zdanavičius, K., \& Straižys, V. 2001, Baltic Astron., 10,349

Zdanavičius, J., Zdanavičius, K., \& Straižys, V. 2005, Baltic Astron., 14, 31

Zdanavičius, J., Čepas, V., Zdanavičius, K., \& Straižys, V. 2010, Baltic Astron., 19,197 